\title{
La disparition de la sidérurgie dans la Volta Region (Ghana)
}

\section{Alain Macé}

\section{(2) OpenEdition}

1 Journals

Édition électronique

URL : https://journals.openedition.org/tc/97

DOI : $10.4000 /$ tc. 97

ISSN : 1952-420X

Éditeur

Éditions de l'EHESS

\section{Édition imprimée}

Date de publication : 1 avril 2004

Pagination : $23-41$

ISSN : 0248-6016

\section{Référence électronique}

Alain Macé, "La disparition de la sidérurgie dans la Volta Region (Ghana)», Techniques \& Culture [En

ligne], 42 | 2004, mis en ligne le 06 novembre 2007, consulté le 29 septembre 2022. URL : http:// journals.openedition.org/tc/97 ; DOI : https://doi.org/10.4000/tc. 97

Ce document a été généré automatiquement le 29 septembre 2022.

Tous droits réservés 


\title{
La disparition de la sidérurgie dans la Volta Region (Ghana)
}

\author{
Alain Macé
}

Depuis la fin du XIXe siècle, la métallurgie africaine retient diversement l'attention. Il serait illusoire de prétendre, en quelques lignes, mentionner l'ensemble des études sur le sujet. Retenons cependant les principaux axes de recherche : les aspects sociaux de cette activité, ses rapports avec la sphère politico-économique, avec la symbolique et la magie et enfin, avec la culture matérielle. Tout en mêlant ces différents registres, un certain nombre de travaux engagent aussi un fructueux dialogue entre ethnologie et histoire : pensons notamment à ceux de Nicole Échard (1986) ou encore à ceux de M-C. Dupré et de B. Pinçon (1997) qui s'appuient sur l'archéologie. Ils contribuent de la sorte à ouvrir le champ de la réflexion sur la technique en général dans les sociétés africaines. L'interdisciplinarité permet donc d'appréhender le travail des métaux sous un nouvel angle, en soulignant la nécessité de ne pas disjoindre les savoir-faire de l'histoire des hommes qui les mobilisent. Ainsi se prémunit-on davantage du risque d'aboutir à des conclusions hâtives, voire parfois discutables. Sur le point très précis de l'extinction des sidérurgies locales, nous sommes invités à recueillir des éléments capables de fournir un éclairage sur les causes de la dépendance de certaines régions bien avant l'aventure coloniale. Par exemple sur la Côte de l'or et sur celle des Esclaves, le secteur que j'ai étudié, le succès du fer européen, loin d'être traduisible en termes d'une quelconque supériorité, fut au contraire la conséquence directe -longtemps ignorée- de multiples crises initiées par les élites de l'Akwamu puis de l'Ashanti; leurs politiques eurent en effet un impact désastreux sur la sidérurgie de la Volta Region. Il faut toutefois se montrer prudent. Le phénomène observé ici ne saurait conduire à énoncer une règle générale, sauf pour souligner la nécessité de toujours retenir le caractère spécifique de tel ou tel contexte. C'est sans nul doute le point sur lequel il convient d'insister, à savoir se garder des extrapolations hasardeuses.

La mémoire

2 Lorsque dans les années 1897-1898, l'ingénieur Fr. Hupfeld (1899 : 174-194) se rend chez les Akpafu, les Santrokofi et les Lolobi, trois populations parmi d'autres installées dans 
la partie montagneuse de la région (carte 1), la sidérurgie y a presque totalement disparu. Hormis quelques rares fours encore en état de marche, le gros de cette industrie appartient désormais au passé. Elle avait pourtant connu un développement tel qu'elle fournissait régulièrement le sud et une partie du centre du Togo (Hupfeld 1899), jusque vers la fin du XVIIe siècle. Sa réputation avait même franchi l'Amu (la Volta), Tokor et Kpandu jouant aussi à cette époque le rôle de places commerciales très actives pour la diffusion du fer. Avant d'analyser les raisons de ce déclin, arrêtons-nous sur les témoignages disponibles.

En 1958-1959, au cours de sa collecte d'éléments culturels sur les "sites abandonnés », le pasteur H.W. Debrunner (1969-1970: $\left.\mathrm{n}^{\circ \mathrm{s}} 10,11,12,13,14\right)$ relève à de multiples reprises la présence de vestiges attestant la pratique -visiblement intense- d'une ancienne activité métallurgique. Des scories jonchent le sol sur de larges étendues en pays avatimé, nkonya, santrokofi, akpafu... Très souvent, les sites abritent aussi des ruines de fourneaux et de forges, des fragments de tuyères ainsi que des enclumes. Enfin, de simples trous et des mines témoignent de travaux d'extraction. Autant de traces que recoupent les traditions orales, comme j'ai pu encore le constater lors d'un séjour à Kpandu en 1997.

Fr. Hupfeld l'avait déjà indiqué sans toutefois y prêter une attention particulière, le secteur alimentait manifestement une vaste zone. L'activité devait d'ailleurs être lucrative et, au moins pour cette raison, le souvenir de tensions inter-sociétales persiste parfois dans la mémoire collective.

Carte 1. Les groupes montagnards de la région de la Volta et la diffusion du fer local à la période précoloniale

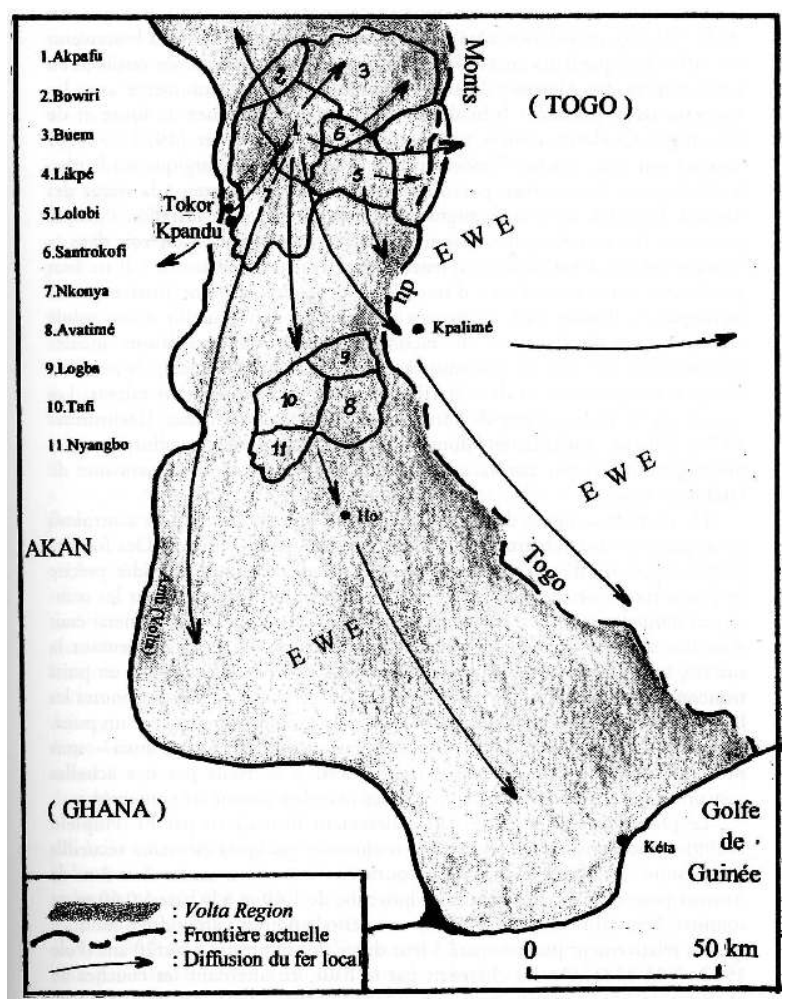

Lors de son passage dans le Litimé au début des années 1950, R. Cornevin (1955 : 21-65) avait de son côté recueilli de la bouche des Akposso le souvenir des difficultés que leurs 
ancêtres rencontraient pour se fournir en outils et en armes auprès des Akpafu: détenant le monopole de ce commerce avec les Akposso, ils se gardaient bien de divulguer leurs techniques de fonte et de façonnage. Quelques années plus tard, H. W. Debrunner (1970 : 75-96) notera à son tour, à la fois l'importance de l'industrie métallurgique sur le plan économique et des conflits sporadiques inhérents, entre autres, à la venue des Akpafu. En effet, ceux-ci se joignirent à des groupes déjà installés. Puisque parmi eux des métallurgistes opéraient avant leur arrivée, doit-on voir dans la relation des incidents les conséquences d'une concurrence accrue? Il ne faut pas écarter cette hypothèse : d'une part, les Akpafu durent finalement se regrouper et, d'autre part, ils purent à juste titre s'enorgueillir d'une solide réputation de fondeurs et de façonneurs. Selon les traditions locales d'Akwamufié (au sud de Kpandu), à une époque non précisée, ils auraient séjourné temporairement dans la ville, exposant de la sorte leurs talents. Les scories sur la place principale sont rapportées à leur résidence (Debrunner 1970). S'ils ne constituèrent donc pas le seul groupe de métallurgistes des montagnes, il est par contre admis qu'ils contribuèrent au dynamisme de l'industrie locale.

6 De nombreux signes d'activité minière ont été repérés en pays santrokofi et en pays nkonya (Debrunner 1970). Ce sont là des exemples. Des fouilles systématiques sur d'autres territoires permettraient de se faire une idée précise de l'importance de l'extraction. Les sites connus nous informent sur les techniques employées, au demeurant rudimentaires. La recherche du minerai était effectuée soit par grattage du sol, soit en prélevant des roches affleurant la surface, soit encore, procédé en apparence plus répandu, en creusant un puits tronconique vertical. Nous y reviendrons. Ces techniques indiquent toutes les limites des moyens disponibles, y compris celle mobilisée pour créer un puits. Les mines des Santrokofi -fort probablement des autres groupes aussi- sont profondes de 5 à $7 \mathrm{~m}$ et sans boiseries. On y accédait par des échelles constituées de troncs d'arbres dont certaines branches avaient été conservées.

7 Le procédé de fonte a été particulièrement bien décrit par Fr. Hupfeld (1899). Procédons à un bref rappel en ajoutant quelques éléments recueillis par la suite. De forme conique, les fourneaux mesuraient entre 2 et $3 \mathrm{~m}$ de hauteur pour un diamètre variant en moyenne de $1,40 \mathrm{~m}$ à la base à $0,60 \mathrm{~m}$ au sommet. Leur fabrication s'étalait sur une période pouvant atteindre 6 mois, ce qui est relativement peu comparé à leur durée de vie prévue pour 20 ans (Pole 1982: 503-513). On les chargeait par le haut, en alternant les couches de charbon de bois et celles de minerai préalablement concassé. $\mathrm{Au}$ terme du processus, l'ouverture inférieure obstruée à l'argile au départ était brisée afin de sortir la loupe. Comme dans le cas des fourneaux de Bassar - un autre site sidérurgique, situé au nord-Togo-, grâce à des orifices (ouverts ou bouchés) situés près de la base, la combustion demeurait sous contrôle. Il n'y avait pas ici non plus de soufflerie mécanique, la ventilation étant assurée par un vent coulis. Notons-le, cette technique très efficace autorisait une économie de main-d'œuvre non négligeable : un seul homme suffisait pour superviser l'opération de fonte. En fait, cette méthode directe de production de fer, dite catalane, n'aboutissait pas à une fonte du minerai à proprement parler : avec une température comprise entre $900 \mathrm{C}$ et $1150 \mathrm{C}$ (inférieure à la température de fusion du fer pur, $1550 \mathrm{C}$ ), on obtenait une masse chargée d'impuretés qu'il fallait ensuite éliminer par des cinglages successifs à la forge. La qualité du produit fini dépendait donc d'un traitement complémentaire. D'une loupe de $4,5 \mathrm{~kg}$ à $24 \mathrm{~kg}$ on extrayait de 3 à $16 \mathrm{~kg}$ de fer utilisable (Pole 1982). Sous l'angle de la 
durée du travail jusqu'à la sortie du fourneau, il n'y a pas de témoignages. De surcroît, nous ne disposons d'aucun paramètre susceptible d'offrir un éclairage précis. L. M. Pole propose la répartition suivante : $60 \%$ du temps pour la préparation du charbon de bois et $40 \%$ pour la fonte. Cette estimation n'inclut ni la collecte du minerai ni celle du bois.

8 Les forgerons produisaient des objets de différentes tailles, de la pointe de flèche à l'outil aratoire. Dans une planche, le capitaine R. S. Rattray (1916: 431-435) mentionne les principaux instruments utilisés : deux soufflets en peau et leurs tuyères en argile, une lame recourbée pour réunir ou étaler les braises, des pinces, un percuteur constitué d'une seule masse de fer, des enclumes en pierre de différentes tailles et un burin. Les pinces traditionnelles resteront en usage jusqu'au début du XXe siècle. Les soufflets étaient fixés sur une base en bois et ils ventilaient le foyer installé à même le sol. Le forgeron travaillait par conséquent en position assise. Outils sacrés, les enclumes faisaient l'objet d'un culte, qui se poursuivra après leur remplacement par des enclumes métalliques (Debrunner 1970: 7-33). Afin peut-être de se protéger des regards indiscrets ou, plus globalement à des fins de défense, des pierres disposées en carré formaient un mur autour des ateliers. L'habitude de construire une enceinte disparut dès que les métallurgistes partirent s'installer dans la plaine au début de la colonisation.

9 Nous sommes ici en présence d'une sidérurgie dont seuls des vestiges et des bribes de traditions orales rendent désormais compte sur le terrain. De l'observation des premiers, il ressort que, au niveau de la technique, cette industrie n'avait rien à envier à celle de Bassar qui réussit, elle, à se maintenir jusqu'au XXe siècle. En tout point comparable quant aux procédés mis en œuvre, elle était a priori promise à perdurer. Il faut y insister, son extinction ne peut pas être rapportée à un différentiel technique. Ainsi l'enquête doit-elle s'inscrire dans une perspective historique. Car alors, comment expliquer, si ce n'est à travers une étude du passé précolonial de la région, la perte de savoir-faire très longtemps aptes à soutenir la concurrence européenne? Mais n'anticipons pas et procédons à l'examen des arguments exposés par le colonisateur. Les éléments d'ordre technique y sont omniprésents, néanmoins dans une perspective telle qu'on ne saurait objectivement y souscrire.

La perspective coloniale

10 La tâche de Fr. Hupfeld consiste à établir un état des lieux des sidérurgies et du potentiel minier du Togo allemand. Sa mission le conduit donc dans la région de la Volta et, au nord, en pays bassar. Même si la présente étude se focalise sur le sud, rien n'interdit de poursuivre si nécessaire nos comparaisons avec le nord, à condition toutefois de prendre en compte des approches se démarquant de celle retenue ici à propos des deux secteurs.

11 À l'issue de son enquête dans la partie méridionale Fr. Hupfeld conclut que ce sont les mauvaises conditions d'extraction du minerai, l'énorme consommation de bois à l'origine d'un prix de revient élevé et enfin, la mauvaise qualité du fer obtenu qui ont entraîné le déclin de la sidérurgie locale. Ces causes avancées appellent une première série de remarques.

12 Sur le plan technique tout d'abord, pas davantage ici qu'en pays bassar (Doering 1894: 48-68) les mineurs n'avaient connaissance de la dynamite, malgré un commerce très actif de la poudre avec l'introduction des armes à feu par les Européens; cela limitait inévitablement les capacités d'extraction. Une fois les gisements épuisés à l'aide des moyens disponibles -en l'occurrence les seuls outils façonnés à la forge-, les mineurs 
devaient partir à la recherche de nouveaux sites. À supposer que le potentiel accessible du secteur ait été épuisé -ce qui ne semble pas être le cas puisque Fr. Hupfeld observe encore quelques opérations de fonte-, il faut alors s'étonner que ni les Akpafu, ni les Santrokofi, ni les Lolobi n'ait quitté les lieux. À propos des Akpafu, cela est d'autant plus curieux que leur tradition orale mentionne une série de migrations (Debrunner 1970: 75-96) dont la trace géographique correspond aux vestiges de métallurgie trouvés entre Cape-Coast et la région de la Volta (Davies $1967: 212$ ). Si aucun indice ne permet avec certitude d'expliquer ces mouvements (fer devenu trop rare ou conflits), en revanche, la résidence prolongée auprès d'autres métallurgistes atteste l'existence de ressources ferrifères suffisantes. La chute de l'activité sidérurgique n'est donc pas imputable à des obstacles techniques de cet ordre.

Il faut ensuite se demander si l'on peut valablement établir une relation de cause à effet entre le volume de bois nécessaire à la conduite du procès de fonte et son déclin. D'autres paramètres entrent en ligne de compte, d'abord ceux relatifs à la présence du fer introduit par les troqueurs; un fait lourd de signification à la fois par sa durée et par son ampleur. Il faut le noter, au début de la traite atlantique, l'Europe n'apporte à l'Afrique rien de plus que ce qu'elle produit déjà (Thornton 1998: 44). Mais, fait curieux, très tôt les métaux (sous forme de barres, de manilles ou d'objets manufacturés) occupent une place de choix dans les échanges, la seconde après les étoffes (Thornton 1998 : 45). Révélateur de pénuries chroniques, ce phénomène n'est pas propre à une zone particulière. J. Rivallain (1994: 37) précise qu'il s'étend à l'ensemble des côtes de l'Afrique de l'Ouest dès le XVIe siècle. Ainsi le simple jeu de l'offre et de la demande a-t-il entraîné une hausse du prix du fer importé. Le fait est d'autant plus curieux que les Hollandais et les Anglais, les deux principaux importateurs, vendent très longtemps de la matière d'œuvre de piètre qualité comparée à celle de son équivalent local (Thornton $1998: 45)$. Hormis dans les régions éloignées des centres sidérurgiques, les importations (comme par exemple au sud de la région de la Volta) répondent par conséquent à une demande née d'une crise de la production. Nous le verrons à propos du centre sidérurgique de la Volta, la résidence des populations dans les montagnes à des fins de défense a influé sur l'écoulement de la production sidérurgique; au point d'entraîner un ralentissement de l'activité, de sorte que la question du volume de combustible ne s'est jamais posée, pas plus que son corrollaire, la hausse de son prix. On peut d'ailleurs douter de la pertinence du critère écologique avancé aussi, de façon générale, par C.Goucher (1981: 179-189) afin d'expliquer la disparition des sidérurgies africaines. Une fois de plus, le pays bassar offre un contre-exemple intéressant: si la fonte n'y joua plus un rôle économique majeur après 1920 , ce fut moins à cause de la rareté du combustible qu'à cause du prix du métal importé (Hahn 1997 : 12). Le problème de la déforestation surgit bien plus tard, après 1950, lorsque le colonisateur s'émut des effets de l'activité sur l'environnement au point de l'interdire (Hahn 1997 : 11). Antérieur au XXe siècle, le déclin de la sidérurgie dans la région de la Volta ne peut pas non plus être rapporté à la consommation de bois. Par suite la question du prix de revient qui lui serait connexe est sans objet.

14 Enfin, le dernier argument, relatif à la qualité du fer, présente une fragilité extrême. Sans doute à la fin du XIXe siècle le métal importé possède-t-il, sinon toujours du moins souvent, des caractéristiques le rendant attractif. Il n'en fut pas toujours ainsi. Pendant très longtemps, le fer local conserve la faveur des Africains (Pole 1982: 503-513; Goucher 1990 : 200-208); à Bassar, par exemple, on accepte de le payer six fois plus cher 
que le métal importé (Pole 1982). Dans cette région comme au sud-Togo, le minerai recèle des traces de manganèse, un facteur important pour la dureté du matériel. Ses principales qualités (malléable et cependant dur) en font un matériau supérieur au fer importé dont la teneur en carbone (supérieure à $1 \%$ ) accroît certes sa dureté mais le rend cassant. Avant l'introduction de «l'acier technique » (moins de $1 \%$ de carbone), le fer local est le seul capable d'offrir une bonne longévité aux outils. Il répond aux attentes des populations, en particulier à celles des agriculteurs. Hormis dans les régions très éloignées des centres sidérurgiques, le fer européen a pu apparaître compétitif en termes de prix, abstraction faite, outre le coût du transport, de sa qualité (Pole 1982; Goucher 1990). Toutefois cette compétitivité reste discutable puisque, selon les estimations de J. Thornton (1998: 47) concernant la Sénégambie, un fort taux de casse et une usure rapide obligeaient à accroître la fréquence des importations. Le fer africain n'avait donc rien à envier à celui venu d'Europe jusqu'à la fin du XIXe siècle. Il faudra attendre la colonisation pour voir arriver sur les côtes un métal attractif. Avant cela, l'emploi du fer importé constitue manifestement un pis-aller. La qualité du fer local n'est donc pas en cause dans le déclin de la sidérurgie dans la Volta région. Le paramètre de la sécurité est en revanche déterminant. Les conflits nuisent au commerce, de fait à l'exportation du fer; en amont, ils compromettent la prospérité des fondeurs au point de perturber, voire d'étouffer l'activité. Ainsi, les hauts et les bas de l'industrie locale doivent être mis en relation avec une instabilité chronique.

15 Aucun des critères retenus par Fr. Hupfeld ne permet de comprendre le déclin prématuré de la sidérurgie de ce secteur. On attenterait néanmoins un mauvais procès à cet auteur en lui reprochant de ne pas engager son analyse dans une perspective historique. Car, ce serait l'oublier, sa mission consiste à évaluer une facette du potentiel de la jeune colonie. Il n'a pas pour objectif de jouer la carte de l'industrie autochtone. D'ailleurs, la teneur en fer du minerai $(54,88 \%)$ (Hupfeld 1899) ne prédispose guère l'Allemagne à relancer, en la développant cette fois-ci, l'activité minière. Ses intérêts se situent sur un autre plan, à savoir fournir à son industrie de nouveaux débouchés. Dans cette optique, il serait absurde de promouvoir la concurrence. Au contraire, toute initiative susceptible d'en interdire l'émergence est la bienvenue, quitte à prendre le risque de créer des mouvements de révolte parmi les populations (Klose 1992 : 187-190). Hupfeld n'en fait pas mystère, l'avenir de l'industrie locale repose désormais sur son aptitude à travailler les métaux importés (fer, cuivre...) et sur la capacité des artisans à s'engager dans des activités de maintenance. Dès lors, on saisit mieux l'orientation de son analyse. Toutefois, répétons-le, s'il avait adopté une démarche historique, il eût été dans l'impossibilité de s'en tenir à la supériorité de l'industrie européenne. Il eût plutôt déduit sans peine que le naufrage de cette sidérurgie résulta des crises aiguës qui bouleversèrent les sociétés locales.

Ni l'étude de Fr. Hupfeld ni, plus tard, celle du capitaine R. S. Rattray (1916: 431-435) qui n'apporte aucun élément nouveau au sujet des Akpafu- n'établissent une relation entre la situation géographique des sites industriels et les populations qui les animent. Il convient pourtant de l'établir. Elles firent de leur habitat un moyen de défense contre les invasions. À la fin du XIXe siècle, l'exposé du lieutenant R. Plehn (1892: 43-60) souligne à leur sujet une singularité sur le plan architectural : des fortifications ont été dressées autour des villages. Visibles nul part ailleurs dans la région, elles confirment l'existence d'un passé tumultueux. Cet indice nous permet d'entreprendre l'analyse des événements auxquels furent mêlés les sidérurgistes. 
Les germes du déclin

17 L'habitat des groupes montagnards est commode à défendre, mais peu propice à l'agriculture et difficile à approvisionner en eau. Toutefois l'avantage principal l'a-t-il emporté sur les inconvénients puisque les États militaires d'Akwamu et de KwawuDudoman ne parvinrent jamais à les soumettre et encore moins à les absorber (Debrunner 1969: 55-78). À la veille du XXe siècle, soit après l'instauration de la pax germanica, ces populations se distinguent toujours par leur indépendance. Hupfeld les qualifie de "inamicales» (undfreundlich), de "craintives» (scheu) et, au sujet des Akpafu, de "passablement louches » (verrufene Leute). Semblables propos, où se mêlent constat et jugement de valeur, ignorent le fait qu'elles eurent souvent maille à partir avec un voisinage turbulent, pour ne pas dire hostile. Et cette donnée essentielle n'apparait toujours pas un siècle après. Ph. de Barros (1986:149-174) attribue la crise de la production sidérurgique que connaît le sud face à la concurrence de Bassar aux XVIIIe et XIXe siècles à la médiocrité de son minerai et à l'absence d'un vaste réseau de diffusion pour ses produits. Ces arguments ne tiennent guère. Bassar bénéficiait certes d'un minerai sensiblement meilleur ( $68,9 \%$ de fer), mais cela n'eut aucune incidence : la métallurgie ne profita pas de la dynamique commerciale des Haousa (Dugast 1992: 675-679). C'étaient les clients qui diffusaient de proche en proche la production; et ce, en fonction des risques encourus selon les périodes de plus ou moins forte instabilité. Jusqu'à la colonisation allemande, elle ne descendit pas au-delà d'Atkpamé (moyenTogo). Von Doering (1894 : 48-68) l'avait déjà constaté, les Bassari ne commerçaient ni de près ni de loin avec la zone côtière. La chute de la sidérur-gie méridionale n'a de fait rien à voir avec Bassar. Cela conforte l'orientation de notre démarche vers des causes extra-techniques.

Du XVIIe siècle au dernier quart du XIXe siècle la région de la Volta est le théâtre d'innombrables conflits (carte 2). Vers la fin du XVIIe siècle, ils secouent le sud -entre Anlo et Bê, Anlo et Fon, Anlo et Guen, Anlo et Ada, Krobo, Akwapim et Ga- de façon quasi permanente. (Cornevin 1969 : 107-108). Cette zone subit de surcroît l'expansion des Akwamu (Gayibor 1990 : 77-78). Après 1702, le nord-est connaît à son tour des flambées de violence : les Akwamu asservissent, toujours selon leurs besoins, des Ewé, les enrôlent parfois de force dans l'armée ou bien les vendent aux négriers (Dantzig 1970 : 221-231). Dès 1710, les Akwamu font de la Traite leur principale activité (Dantzig 1970 : 239-296), semant la terreur dans l'ensemble de la région. La recherche de futurs captifs accroît l'insécurité. De leur côté, les Ashanti ne restent pas inactifs. Entre 1721 et 1744 , leurs assauts répétés repoussent toujours plus vers l'est les Kwawu, au point de les obliger à franchir la Volta et à entrer physiquement en contact avec les Nkonya, les Santrokofi... et les Akpafu. Les Ashanti occupent le devant de la scène, puisque la viabilité de leur empire suppose le maintien de relations tributaires fortes avec les populations périphériques. Ainsi, peu à peu, à l'exception notoire des groupes montagnards, ils placent sous leur autorité directe ou indirecte toutes les populations jusqu'à Petit-Popo. 
Carte 2. Offensives des Akwamu puis des Ashanti et de leurs alliés vers l'est du XVIle au XIXe siècles

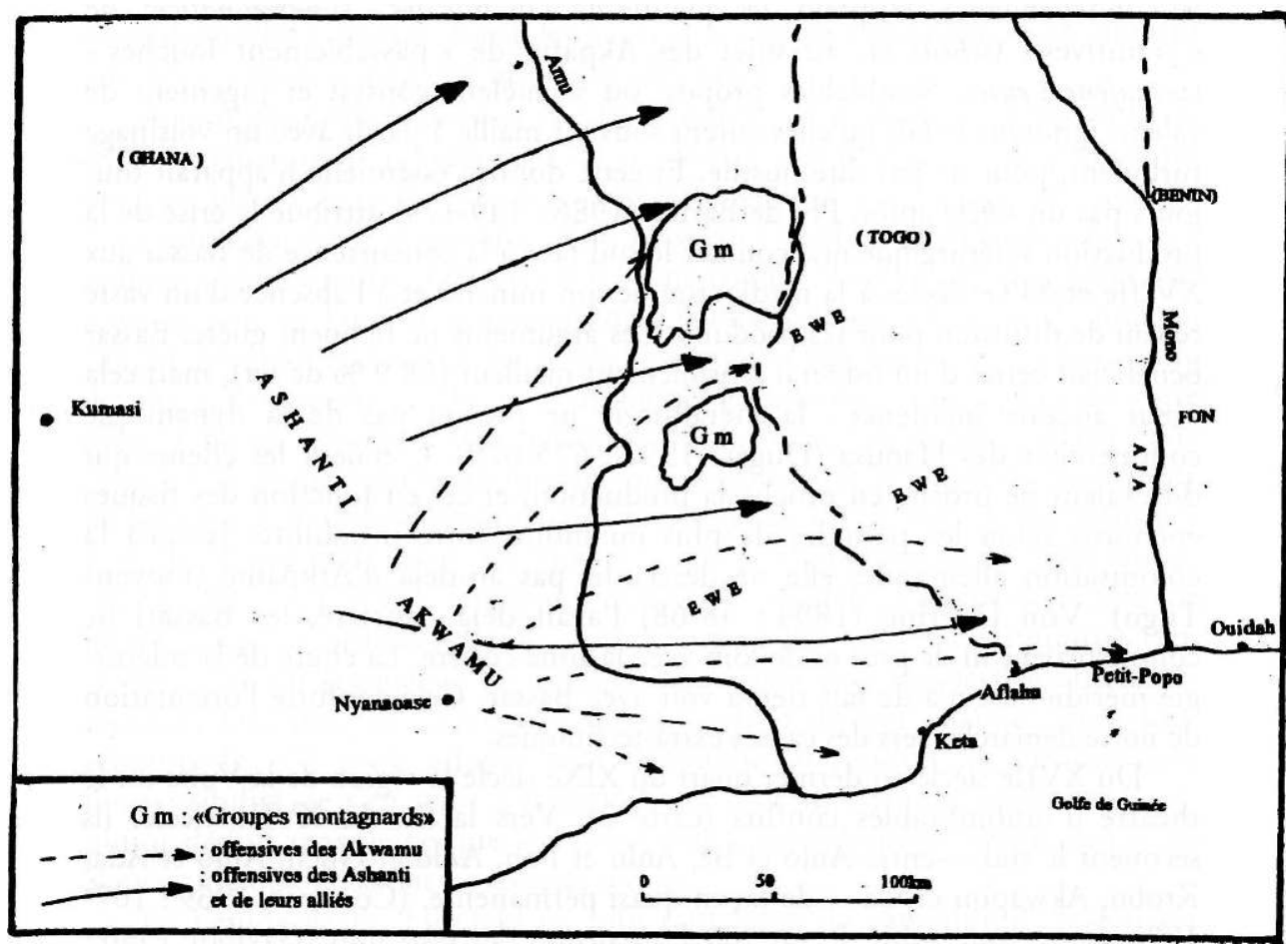

Une aire d'influence aussi vaste présente d'inévitables faiblesses. Elle inspire également la crainte. Pour cette raison le royaume d'Abomey lance une offensive sur le sud en 1764 (Wilks 1975 : 320). Après la victoire des Fon, la zone demeure agitée. Sur la requête des Danois, qui tentent de s'établir durablement à Kéta -et soucieux de faire taire les querelles sporadiques entre les Anlo et Petit-Popo, nuisibles aux affaires-, les Ashanti lancent des expéditions punitives à partir de 1792 (Wilks 1975 : 321-324). En 1818 puis en 1822-1823, c'est au tour des populations du Nkonya et du Wusuta de subir leurs assauts (Wilks $1975: 173,700$ ). Malgré un habitat protecteur, les groupes montagnards ne sont donc pas à l'abri des menaces. Un calme relatif prévaut par la suite, jusqu'en 1826 où des Ewé décident de revendiquer leur indépendance. L'initiative échoue, l'alliance entre les Péki et les Akwamu réussissant à étouffer la rébellion. Plus tard, en 1833, naissent d'autres soulèvements; mais cette fois-ci, ce sont les Anlo qui s'allient aux Akwamu. Les Ashanti décident alors de mettre au pas les Ewé de la Volta (Wilks 1975 : 210-211). De multiples conflits d'étendue variable vont néanmoins persister parmi lesquels celui entre les Akpafu et les Kwawu vers 1850-, en vertu du principe selon lequel un roi ashanti se doit de partir en campagne dans les trois années qui suivent son accès au trône (Terray 1982: 375-421). Les États tributaires sont ainsi rappelés à l'ordre de façon régulière et les sociétés lignagères (en général éwé) constituent toujours des réserves de captifs potentiels. Le négociant M-J. Bonnat en fait l'amère expérience lors du saccage des alentours de Ho par les Ashanti et leurs alliés en 1869 (Perrot \& Dantzig 1994 : 41-166). Secondés par les Anlo et les Akwamu, Kumasi lance en effet des opérations d'envergure sur une vaste zone englobant Tokor, le Buem, l'Aveme, le Tsome, le Tafi, l'Adele (Wilks 1975 : 224-225).

De si nombreux remous ne favorisent guère la régularité des échanges. Dans la mesure où ils influent de facto sur l'écoulement des produits (Debrunner 1969: 54-78), ils perturbent par contrecoup la sidérurgie elle-même. Retranchés dans les montagnes 
afin d'échapper aux razzias, les métallurgistes assistent à l'asphyxie de leur industrie. Des phases de pénurie s'ensuivent nécessairement. Au cours de la première moitié du XVIIIe siècle, l'esclavagiste danois L.F. Romer (Dige-Hess 1989: 92, 143) mentionne dans ses notes de voyage que le fer est devenu plus rare que l'or et qu'il en vaut le prix. Tout le long de la Côte des Esclaves ce sont alors les Danois, les Anglais et les Hollandais qui alimentent pour l'essentiel ce marché. Les factoreries de Keta (au sud de la région de la Volta) et de Ouidah (sur le littoral de l'actuel Bénin) approvisionnent les Akwamu et l'arrière-pays (Kea sd : 371-392). Les Anglais et les Hollandais, les plus impliqués dans ce négoce, n'hésitent d'ailleurs pas à se concurrencer tant la demande est forte. Agent de la Generaele Geotroyeerde West Indische Compagnie, Jan de Paauw insiste régulièrement dans ses rapports sur la nécessité d'importer des quantités massives de barres de fer (Dantzig 1978: 141, 169, 172). À l'appui de sa démarche, il indique l'importance accordée au précieux métal juste avant la saison agricole (Dantzig 1978 : 208). Nous disposons là d'une information capitale : les communautés rurales doivent s'en remettre aux troqueurs pour obtenir la matière d'œuvre utile à la confection de l'outillage. La pénurie touche visiblement une zone étendue car le fer ne reste pas sur la côte; il monte loin à l'intérieur des terres. Tandis qu'ils négocient des achats d'or avec les Ashanti, en 1706-1707, les Anglais introduisent 8000 barres de fer à Ouidah (Dantzig 1978 :197-238). Le phénomène se poursuit au XIXe siècle et peut-être même s'aggrave-til. Les Anglais, par exemple, élèvent le volume de leurs exportations : de 656 tonnes en 1827, elles passent à 3691 tonnes en 1850 (Newbury $1961: 234$ ). En 1869, le négociant M.-J. Bonnat vend aux Ewé de la Volta, parmi d'autres produits, des barres de fer et de la coutellerie (avec un bénéfice de 100\%!) (Perrot \& Dantzig 1994: 45). Jusqu'à la pacification européenne, ce commerce est d'autant plus lucratif que la zone demeure instable.

Depuis la naissance de l'empire des Akwamu au XVIIe siècle, la région de la Volta doit diversifier ses sources d'approvisionnement. Elle fait appel à son industrie sidérurgique lors des périodes de paix et aux troqueurs en temps de conflits. Le recours au fer importé traduit toujours une phase de pénurie. Une dépendance naît de fait très tôt, bien avant que n'interviennent les critères de la qualité et du coût. Elle résulte d'abord de la politique de l'Akwamu puis de celle de l'Ashanti dont les groupes montagnards subissent indirectement l'un des effets.

Le prix des ambitions

21 À l'évidence, cette sidérurgie locale ne retient l'attention ni des Akwamu ni des Ashanti. Comment traduire une telle absence d'intérêt alors que l'accès au fer représente un enjeu stratégique? On pourrait établir un rapport avec la présence du fer introduit par les troqueurs. Mais nous avons déjà vu ce qu'il faut penser de ce matériau. Il reste alors les armes à feu, autre produit très prisé, dont on imaginerait qu'elles ont permis l'engagement des guerres et entraîné le déclin de l'industrie. Ce serait oublier qu'elles constituent un facteur secondaire. Si, par exemple chez les Gonja, les Dagomba et les Mamprusi, les chevaux sont l'apanage de l'aristocratie (Goody 1971 : $36,48,64,71)$, ici où les épizooties interdisent la constitution d'une cavalerie, ce sont au départ les armes d'apparat puis ensuite les fusils qui présentent cette qualité. Il faut par conséquent équiper la troupe qui occupe les premières lignes lors des combats. Nombreuse grâce à un recrutement de masse forcé, cette troupe utilise les armes traditionnelles (lances, arcs...) (Thornton $1998:$ 112-125). Alors, objectera-t-on, c'est donc bien grâce au matériau européen, même médiocre, que les conflits perdurent. Il faut dans ce cas supposer que la sidérurgie de la Volta est la seule disponible; ce qui est 
inexact. La réponse réside dans les dynamiques internes de l'Akwamu et de l'Ashanti dynamiques insufflées et entretenues par leurs élites respectives.

Avant que des luttes intestines et des rapports dégradés avec leurs voisins ne les rendent vulnérables aux pressions des Ashanti vers 1730, les Akwamu connaissent un demi-siècle de prospérité. Grâce aux taxes sur le commerce à longue distance pour l'essentiel, aux surplus agricoles et à l'esclavage (en partie à usage domestique, en partie destiné à la traite), la sphère dirigeante dispose des moyens nécessaires à son entretien (Kea s.d.: 371-392). La richesse acquise permet de maintenir l'intégrité de l'empire. S'il le faut, l'État peut facilement engager des expéditions punitives. Une judicieuse répartition des activités favorise l'équilibre entre le centre historique et sa périphérie. La province qui s'étend autour de Nyanaoase, la capitale, produit une bonne partie de l'alimentation de l'empire, tandis que les autres se spécialisent dans le commerce; un commerce, rappelons-le, lourdement imposé. Pareil mode de gouvernement crée de solides rapports de réciprocité. Ils obligent les groupes soumis à exercer de fortes pressions sur leurs voisins (Kea s.d.: 371-392). Cela explique les razzias à la fin du XVIIe et au début du XVIIIe siècles. Il importe guère aux Akwamu de voir étouffer la sidérurgie des groupes montagnards; ils bénéficient toujours de la production des Akwapim à l'ouest et, au pire, ils détiennent la capacité (en or puis en esclaves) de se fournir auprès des troqueurs. De la sorte, la baisse d'activité de ce secteur n'a aucune incidence fâcheuse sur l'État. Au contraire, elle permet de renforcer les contraintes à l'égard des dépendants. À l'inverse, les sociétés lignagères, celles qui subissent les razzias en raison de leur fragilité, la ressentent de plein fouet. En majorité agricoles -c'est le cas des Ewé de l'arrière-pays-, elles dépendent régulièrement des troqueurs. Au XVIIIe siècle, cela signifie aussi s'impliquer dans la traite (certes à une échelle moindre).

À la suite des Akwamu, les Ashanti mènent une politique tout aussi désastreuse pour la sidérurgie de la région de la Volta. Sa durée et son ampleur auront des conséquences définitives. Elle se distingue néanmoins de celle de leurs prédécesseurs : à la soumission brutale, les Ashanti préfèrent les relations tributaires. Si Nyanaoase avait conduit une politique à bien des égards centraliste, Kumasi opte plutôt pour le fédéralisme. Il n'en demeure pas moins que, là aussi, cela vise la satisfaction des intérêts de l'élite.

Lorsque les guerres, utiles à la création d'un État fort, sont achevées, les Ashanti s'efforcent au XVIIIe siècle d'exercer un étroit contrôle des États périphériques - dont ce qui subsiste de l'Akwamu-, charge étant alors dévolue à ceux-ci de razzier les sociétés lignagères. La stratégie de l'élite consiste à entretenir le rapport tributaire sur le plan extérieur et le rapport esclavagiste sur le plan intérieur. Le premier suppose la supériorité militaire -déjà ancienne mais renforcée par un strict contrôle du commerce des fusils et de la poudre à destination de l'aristocratie (Bowdich 1819 : 335) - et le second, un encadrement du travail servile dont les bénéfices (surtravail et surplus) reviennent à la sphère dirigeante. Au sommet de la pyramide sociale règne une aristocratie avant tout militaire, le roi (asantehene) en étant le chef. Les activités commerciales restant mineures, l'ascension individuelle passe nécessairement par l'art de la guerre; il faut acquérir des mérites sur le champ de bataille afin de justifier son rang, ou en vue de l'améliorer (Terray 1982 : 375-421). L'État entretient cette logique à deux niveaux : en lançant de fréquentes offensives et en veillant à la conservation de rapports tributaires forts qui obligent les États vassaux à razzier les sociétés lignagères, pour répondre à la fois à leurs besoins et à leurs obligations. Purement alimentaires, ces 
guerres esclavagistes sèment la terreur. De fait, elles déstabilisent de façon épisodique les réseaux d'échanges. La politique ashanti va ainsi repousser les Kwawu sur la rive orientale de la Volta, accroissant du même coup les pressions sur diverses populations, dont des groupes montagnards. Les Akpafu s'efforcent de desserrer l'étau qui provoque leur isolement. Ils vendent alors des esclaves maako aux Ashanti (Debrunner 1970: 7-33). L'industrie sidérurgique connaît de sérieuses difficultés puisque des agents de l'asantehene achètent du fer sur la côte auprès des troqueurs dès la première moitié du XVIIIe siècle (Dige-Hess 1989 : 143, 151). Les Ashanti doivent donc parer à des pénuries chroniques de ce matériau, malgré la diversité de leurs sources d'approvisionnement. Lors de son séjour chez eux en 1819, T. E. Bowdich (1819: 185-186) note qu'ils s'en procurent d'ordinaire auprès du Gyaman, du Gonja et du Dagomba. Remarquons-le, le centre sidérurgique de la Volta n'est pas mentionné. Il ajoute qu'ils revendent du fer acquis sur la côte aux Dagomba, ces derniers le "préférant » à celui produit sur place (Bowdich 1819: 446). Il faut s'arrêter sur ce que recouvre en réalité cette "préférence ». T. E. Bowdich ignore ou bien oublie de mentionner un fait essentiel : la dégradation des rapports entre les Dagomba et les Bassari a entraîné une baisse de la production sidérurgique à l'ouest du pays de ces derniers à la fin du XVIIIe siècle (Barros 1986 : 149-174), production dont les premiers dépendent pour répondre à leurs besoins propres et pour satisfaire à leurs diverses obligations de tributaires vis-à-vis des Ashanti. Par conséquent, on peut à juste titre douter que cette " préférence » ait été imputable, comme le croît T. E. Bowdich, à la qualité du fer européen, surtout si l'on garde en mémoire que le fer des Bassari conservera jusqu'au XXe siècle la faveur d'une vaste clientèle. Le métal vendu auparavant aux Dagomba est alors nécessairement un fer brut de four -ce qui explique le propos de Bowdich-, à savoir devant être épuré avant d'être façonné. Cela ne confère pour autant aucune qualité supplémentaire au fer importé. Par ailleurs, la vente du précieux matériau européen en petites quantités et à un prix élevé (Bowdich 1819: 457) n'a rien d'étonnant : l'objectif de Kumasi consiste à permettre aux Dagomba de continuer à jouer leur rôle de tributaires en fournissant aussi des produits finis (des lames de couteaux, de houes, des récipients...), pas de les voir s'émanciper (Bowdich 1821: 36). Au XIXe siècle, les Ashanti doivent de surcroît tenir compte de l'abolition de la traite négrière décrétée par les Anglais en 1807, sans néanmoins renoncer à leur suprématie. Même si une traite clandestine subsiste, il devient désormais impossible de faire de la recherche de captifs un des grands axes de la politique (Wilks 1975). Il faut plutôt la réorienter vers le commerce. Les produits venus de la côte et la noix de kola à destination des Haousa représentent des taxes substantielles. Tout cela suppose une réforme des institutions, qui n'aboutira pourtant pas. Une surpopulation d'esclaves constitue certes une sérieuse menace pour la stabilité de l'État, mais les tributaires qui en sont les principaux pourvoyeurs risquent de se rebeller. Un exemple parmi d'autres, la campagne de 1869-1870 contre la Volta region se solde par des déportations massives (Wilks 1975 : 84-86). Toute la politique ashanti du XIXe siècle tourne autour d'un compromis visant à satisfaire et les impérialistes et les mercantilistes parmi les dirigeants (Wilks 1975: 482, 477-549). Grand producteur de kola, l'empire se soucie de garantir la sécurité des routes du nordest et donc d'éviter les guerres à tout prix. Le commerce entre les Haousa et leurs clients sur lequel ils perçoivent des taxes en dépend. En revanche, afin de ménager les tributaires qui se convertissent petit à petit à la traite des produits, l'empire ne se préoccupe pas de l'instabilité de la région de la Volta. Au contraire, elle lui est profitable; plus les conflits y perdurent en vue d'acquérir davantage de main-d'œuvre 
domestique et, accessoirement, des captifs destinés à la traite illégale, plus les troqueurs importent de marchandises sources d'impôts : fusils, récipients, coutellerie... et barres de fer. Les moyens considérables (économiques et militaires) dont disposent toujours les Ashanti, les dispensent de s'inquiéter des aléas de l'industrie chez quelques groupes retranchés dans leurs montagnes. Après la campagne militaire de 1869-1870, l'arrêt de la navigation sur l'Amu et le blocus imposé aux Ewé-Krépi portent un rude coup aux échanges locaux. Face aux menaces quasi permanentes, les Akpafu, les Santrokofi... réduisent leur activité et s'inquiètent d'assurer davantage leur indépendance sur le plan alimentaire. L'agriculture de subsistance se substitue ainsi encore un peu plus à la sidérurgie.

Il va falloir attendre l'effondrement de l'empire ashanti sous les coups des Anglais (1874) puis l'arrêt des multiples guerres tribales (1890) après l'intervention allemande, pour qu'enfin une paix durable soit rétablie. Sauf quand ils sont devenus des lieux de culture, les groupes montagnards abandonnent leur village de montagne et s'installent dans la plaine (Debrunner 1969). Mais il est désormais trop tard pour relancer la sidérurgie. Les réseaux de commerce du fer ne sont plus axés sur l'industrie indigène, loin de là. À l'inverse du pays bassar qui est toujours resté à l'abri des importations européennes (Doering 1894), le Sud-Togo, lui, compose déjà avec elles depuis au moins deux siècles. Elles ont indirectement contribué à la longue agonie de son industrie. La colonisation portera le coup de grâce.

La disparition de la sidérurgie dans la Volta region résulte de façon très nette des politiques expansionnistes de l'Akwamu puis de l'Ashanti entre le XVIIe et le XIXe siècles. Les dynamiques internes de ces États supposent des pratiques prédatrices, promises à perdurer d'autant plus qu'elles fournissent les moyens d'ignorer les dommages qu'elles induisent. Il faut donc bien appréhender sous l'éclairage des rapports inter-sociétaux les incidences sur une industrie que rien, a priori, ne prédispose à une extinction prématurée. Une étude centrée sur la présence européenne en tant que cause aboutit à un contresens, car le fer importé ne possède pas les qualités requises permettant de le substituer au fer local. Seules les guerres, en provoquant des pénuries chroniques, justifient son introduction. Elles perturbent les échanges au point de rendre impossible la satisfaction régulière des attentes à partir des ressources indigènes. Par contrecoup, les métallurgistes n'ont d'autre choix que de réduire la production; et ce, jusqu'à lui conférer un caractère anecdotique. La sidérurgie des groupes montagnards ne souffre pas, c'est clair, de «faiblesses » imputables aux savoirfaire mobilisés. Aussi doit-on définitivement bannir les perspectives condamnant la mémoire au silence. Ici comme ailleurs l'histoire des techniques se confond avec celle des hommes. 


\section{BIBLIOGRAPHIE}

Barros, Ph. de

1986. «Bassar : a quantified, chronologically controled, regional approach to a traditional iron production centre in West-Africa ", Africa 56 (2) : 149-174.

Bowdich, T. E.

1819. Voyage dans le pays d'Ashantie ou Relation de l'ambassade envoyée dans ce royaume par les Anglais. Paris : De Gide Fils.

1821. An Essay on the Superstitions, Customs and Arts Common to the Ancient Egyptians, Abyssinians and Ashantes. Paris : J. Smith.

Cornevin, R.

1955. « Le Litimé », Études Dahoméennes (IFAN) XIV : 21-65.

1969 Histoire du Togo. Paris : Berger-Levrault (1ère édition 1959)

Dantzig, A. van

1970. Les Hollandais et 1 'essor de l'Ashanti et du Dahomey. Thèse de 3ème cycle, Paris : EPHE. 1978. The Dutch and the Guinea Coast, 1674-1742. A collection of documents from the general state Archives at The Hague. Accra : GAAS.

Davies, 0 .

1967. West Africa before the Europeans : Archaeology and Prehistory. Londres : Methuen.

Debrunner, H.W.

1969-70. « Notes sur les peuples témoins du Togo, à propos de sites montagneux abandonnés ", Bulletin de l'Institut d'Enseignement Supérieur du Bénin, 10-11-12-13.

Dige-Hess, M.

1989. Romer, L.F. Le golfe de Guinée, 1700-1750. Paris : L'Harmattan.

Doering, Lt G. von

1894. « Voyage de Bismarckburg par Fasugu jusqu’à Bassari (du 26 mai au 15 juin 1894)», traduction française ronéotypée. Lomé : Université du Bénin.

Dugast, S.

1992. Rites et organisation sociale : l'agglomération de Bassar au Nord-Togo. Thèse. Paris : EHESS (3 vol.).

Dupré, M.-C. \& B. Pinçon

1997. Métallurgie et politique en Afrique centrale. Deux mille ans de vestiges sur les plateaux batéké.

Gabon, Congo, Zaire. Paris : Karthala.

Échard, Nicole

1986. « Histoire du peuplement et histoire des techniques : l'exemple de la métallurgie hausa du fer au Niger », Journal des Africanistes 56, $1: 20-32$.

Gayibor, N.L.

1990. Le Genyi. Un royaume oublié de la côte de Guinée au temps de la traite des Noirs. Lomé : HahoKarthala.

Goody, Jack

1971. Technology, Tradition and the State in Africa. Londres : Oxford University Press. 
Goucher, C.

1981. «Iron is iron 'til it is rust », Journal of African History XIII : 179-189.

1990. «African hammer, European anvil : West African Iron Technology in the Atlantic Trade Era ", pp. 200-208, in Cultural Ressource Management : An African Dimension, Bassey W. Andah Wisdom Publishers Ltd, West African Journal of Archeaology, Ibadan Owerri.

Hahn, H. P.

1997. Techniques de métallurgie au Nord-Togo. Lomé : Presses de l'Université de Lomé.

Hupfeld, Fr.

1899. «Die Eisenindustrie in Togo », Millteilungen aus den deutsche Schutggebieten 11 :175-194.

Kea, R. A.

Sd. « Administration and Trade in the Akwamu-Empire, 1681-1730 », EHESS, Fond Brunschwig, pp 371-392.

Klose, $\mathrm{H}$.

1992. Le Togo sous drapeau allemand, 1894-1897, selon Henrich Klose. Lomé: Haho-Karthala (« Les chroniques anciennes du Togo ») (1ère édition 1899, Berlin : « Togo unter deutscher Flagge »).

Newbury, C. W.

1961. The Western Slave Coast and its rulers. European Trade and Administration among the Yoruba and Adjà-Speaking Peoples of Southern Dahomey and Togo. Oxford : Clarendon Press.

Perrot,C.-H. \& A. Dantzig (van)

1994. Marie-Joseph Bonnat et les Ashanti - Journal (1869-1874). Paris : Mémoires de la Société des Africanistes.

Plehn, Lt. R.

1892. «Beitrage zur Volkerkunde des Togogebietes », Halle (trad. fr : « Avec le lieutenant Plehn à la recherche d'un cercle du Moyen-Togo », in Études dahoméennes, IV, pp 43-60).

Pole, L. M.

1982. «Decline or survival ? Iron production in West Africa from the seventeenth to the twentieth centuries ", Journal of African History XIII : 503-513.

Rattray, R. S.

1916. « The Iron workers of Akpafu », Journal of the Royal Anthropological Institute 46 : 431-435.

Rivallain, J.

1994. Échanges et pratiques monétaires en Afrique du XVe au XIXe siècles à travers les récits de voyageurs. Paris : Musée de l'Homme.

Terray, Emmanuel

1982. " Nature et fonction de la guerre dans le monde akan XVIII-XIXe siècles ", pp. 375-421, in

Guerres de lignages et guerres d'États en Afrique. Paris : Édition des Archives contemporaines.

Thornton, J.

1998. Africa and the Africans in the Making of the Atlantic World, 1400-1800. Cambridge : Cambridge University Press (1ère édition 1992).

Wilks, I.

1975. Asante in the Nineteenth Century. The Structure and Evolution of a Political Order. Cambridge : Cambridge University Press. 


\section{RÉSUMÉS}

Deux causes furent souvent avancées pour expliquer l'extinction des sidérurgies africaines : la présence occidentale et les faiblesses des techniques autochtones. L'Europe a certes exercé une influence considérable. Sans aucun doute de nombreux savoir-faire locaux se virent-ils, à terme, incapables de rivaliser avec ceux issus des révolutions industrielles. Néanmoins, ainsi mis en exergue, le commerce et la technique risquent fort d'apparaître comme des facteurs depuis toujours déterminants. Or, rien ne serait plus inexact. $\mathrm{Si}$, dès le XVIe siècle, des populations de la Volta Region utilisent du fer européen, c'est davantage en raison des rapports inter-sociétaux. Les politiques conduites par les Akwamu puis par les Ashanti portèrent en effet un coup mortel à l'industrie locale.

The disappearing of siderurgy in the Volta Region (Ghana). Two causes were often set to explain the decline of the african siderurgies : the occidental presence and the weaknesses of the indigenous processes. Most certainly Europe exercised a wide influence. There is no doubt that many local techniques were unable to compete, at last, with those born from the industrial revolutions. Nevertheless, when the commercial and the technical aspects are emphasized, they could be considered as obvious evidences. But this would be inacurrate. If some african populations, since the XVIth century, get iron from europe, the motives have to be sought somewhere else. In the Volta Region the reasons for this importation are more likely to be found in the relations between different societies of this region. As a matter of fact, the politics leaded by the Akwamu and then by the Asante dealt a heavy blow to the local industry. It will never rise up again.

La desaparición de la siderurgia en la Volta region (Ghana). Frecuentemente, dos causas se expusieron para explicar la extinción de las siderurgias africanas: la presencia de los Occidentales y la pobreza de las técnicas autóctonas. Desde luego, el influjo de Europa al respecto fue muy importante. Numeros sos saber-hacer locales han sido incapaces de competir con los que nacieron en las revoluciones industriales, no cabe duda. Sin embargo, hacer hincapié de tal modo en el comercio y la técnica puede llevar a pensar que son factores determinantes, y eso desde siempre. Ahora bien, no es así. Cierto es que a partir del siglo XVI algunas poblaciones de la Volta Region emplean hierro europeo. Pero es más bien debido a relaciones interétnicas. Las políticas de los Akwanmu y después, las de los Ashanti, hirieron mortalmente las industrias locales.

\section{INDEX}

Mots-clés : histoire, afrique, Ghana, politique, sidérurgie, Volta Region

\section{AUTEUR}

\section{ALAIN MACÉ}

Docteur en Anthropologie sociale et en Ethnologie (EHESS, Paris) 